\title{
Research on Signal Detection Technology for Flexible Sensor to Monitor the Temperature and Pressure of the Battery
}

\author{
Shufeng.Zhang ${ }^{1, a}$, Ziguan.Zhou ${ }^{2, b}$, Yanbin.Song ${ }^{2, b}$, Shengjie.Zhang ${ }^{3, c}$ \\ ${ }^{1}$ Xinzhou Supply Branch of Shanxi Power Company, Xinzhou 034000, China \\ ${ }^{2}$ Beijing Smartchip Microelectronics Technology Co., Ltd, Beijing 102200,China \\ ${ }^{3}$ China Electric Power Research Institute, Beijing 100192,China \\ aZZF@163.com, blancer101@163.com, 'SJ@163.com
}

Keywords: Battery; Signal Detection; Flexible Sensor.

Abstract: In view of the current situation of the storage battery temperature and deformation monitoring is not satisfactory, a flexible sensor signal detection technique for monitoring the temperature and pressure of the battery is presented. The principle and characteristics of the flexible sensor are introduced, the processing circuit of the temperature pressure signal is designed, and the signal is verified by experiments. The results show that the signal detection technique can be used for the monitoring of the temperature and pressure of the battery.

\section{Introduction}

As one of important issues of electric power system, the battery is able to provide safe, reliable and robust DC power resource. Due to long-term operation under high temperature circumstance, degradation of the battery will be greatly accelerated with worse corrosion and water loss, which would be very dangerous. Moreover, the battery would swell or distort, and these failures may lead to security problem [1]. therefore, detection technology is necessary to make sure the security of the battery.

Currently, there exist various detection approaches. However, these traditional detection technologies usually adopted thermal resistance or semiconductor sensor to conduct temperature monitoring, where the accuracy is hard to satisfied the demand. While temperature sensors based on resistances are not so convenient for application, due to high cost, complexity, and low reliability. Additionally, these traditional MEMS or CMOS sensors are mainly based on the semiconductor technologies, with limited materie and measurements. And those sensors have some common disadvantages, like simple function, great complexity, heavy weight and big power-comsupsion, etc. All those disadvantages make traditional ones unable to meet special demands by electric power equipment or system [2].

Benefiting from advanced nanometer materials technologies, flexible sensors is able to combine various technologies together, such as sensing, detection and communication etc. And flexible sensors will replace traditional ones, which are charactrised by large size, high power-comsumption, and single function. Thus, flexible sensors will meet the demand of electric power mornitoring. Therefore, this work proposes a novel signal detection technology for flexible sensor to monitor the temperature and pressure of the battery. In thits work, the flexible sensor can be simplely attached on the surface of the battery to monitoring the temperature and pressure of the battery with low cost.

\section{Brief Introduction of Flexible Sensors}

The basic idea of the flexible sensor is to sense any change of pressure using pressure-sensitive material [3-4]. And the flexible temprature sensor makes full use of nanometer temprature-sensitive materials to detect the static or dynamic change of temperature [5].

In general, the flexible sensor consists of sensing elements, signal detecting and processing elements, communication element, etc. In the flexible sensor, the sensing element is the core of this kind of sensor and is made of manometer sensing materials. And the signal detecting and processing 
element is responsible of transforming analogy signals into digital ones, while the communication circuit is to conduct sensing, converging and sending operation of both power devices states and environment information. The typical structure of the flexible sensor is depicted in Fig.1.

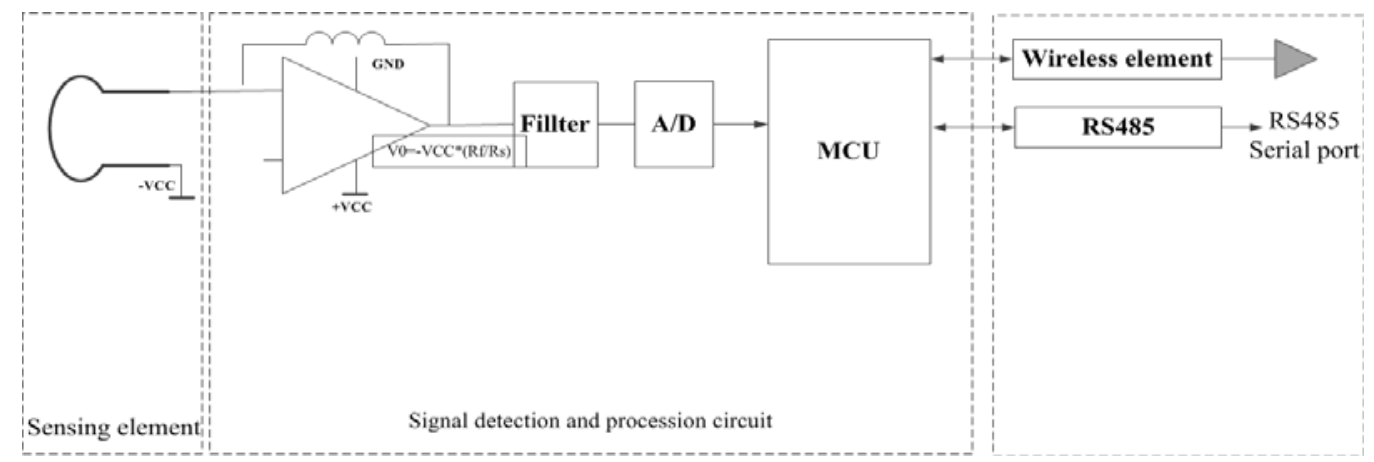

Fig.1 Structure of the flexible sensor

\section{Design of Sensing and Processing Circuit}

Aimed to solve the degradation problem of battery, a novel signal detection approach based on the flexible sensor is proposed and designed to monitor the temperature and pressure of the battery, including the Signal Detection Element and the Signal Processing Circuit.

(1) Signal Detection Element

In the flexible sensor system, the signal detecting element is designed for signal detecting and collecting, in which the "Upper/Lower Module" structure is adopted. And this structure is convenient for development with low cost, simple operation and well-defined functions [6].

The Lower Module is designed as shown in Fig.2, which is in charge of information colletng, data storing and messages reporting. In the informtation collection element of Fig.2, the power supply is $5 \mathrm{~V}$, including 5 temperature sensing elements, 1 pressure sensing element, and 2 pressure-intensivity sensing elements all with frequency of $0.2 \mathrm{~s}$ per time. And the signal receivor transforms the output signal from sensing array into voltage signal, and then amplifys voltage signal. To makesure of accury, the power supply is given accurately and staticlly by the power element. And the Flash is used to record data collected and work with the clock element to mark time that it takes to collect these datas. Later, the wireless communication element will report stored data to the Upper Module.

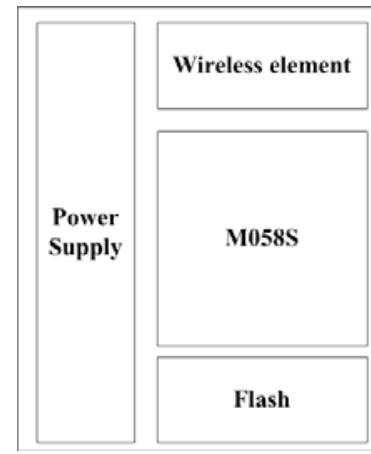

Fig.2 Design of the lower module

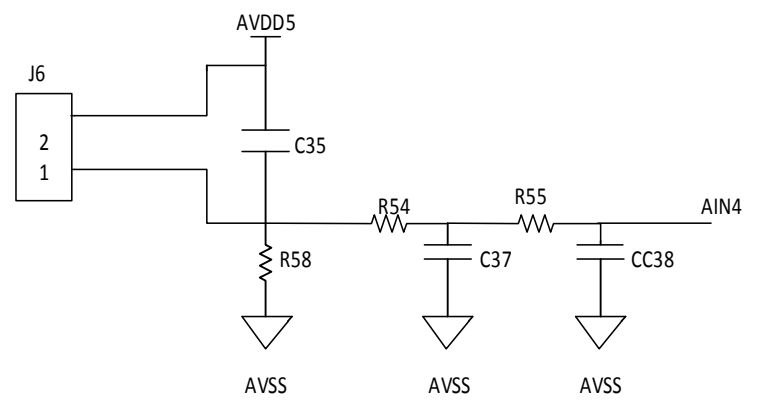

Fig.3 Temprature signal processing circuit

(2) Signal Processing Circuit

The sensor in the collection circuit can be viewed as a variable resistance, and its resistance value will change with the pressure or temprature factors [7]. In the Lower Module, the accuracy is determined by adjusting the load impedance. In fact, the accuracy of sensors will degrade with lower load impedance value, but the detecting range will be enlarged under this condition. Therefore, it Is the key issue to sellect apropriate load resistance [7].

Duiring the procedure of signal detection, it is necessary to input both of pressure-resistance signal and temprature-resistance one into processor. Therefore, it is required to transform both of pressure-resistance signal and temprature-resistance siganl into voltage, and to adjust the voltage by 
amplifier circuit to makesure it is within the range of data collector. Both of temprature signal processing circuit and pressure signal processing circuit are key issues as the core of signal dection and trassmission, which must satisfy high-speed requirement.

The temprature signal processing circuit is given in Fig.3. The AVDD5 works to provide direct power supply for the whole detection procedure, and J6 is the temprature sensor. As the temprature of J6 changes, its resistance value also changes and the one of R59 will be reported to the upper-module after AD transforming. The pressure signal processing circuit is given in Fig.4. In Fig.4, the J7 works as the piezoresistor, and the capacitor C41 is play the role of filter and stabilivolt. the MAX4494 is a dual-stage amplifier shown in Fig.5.

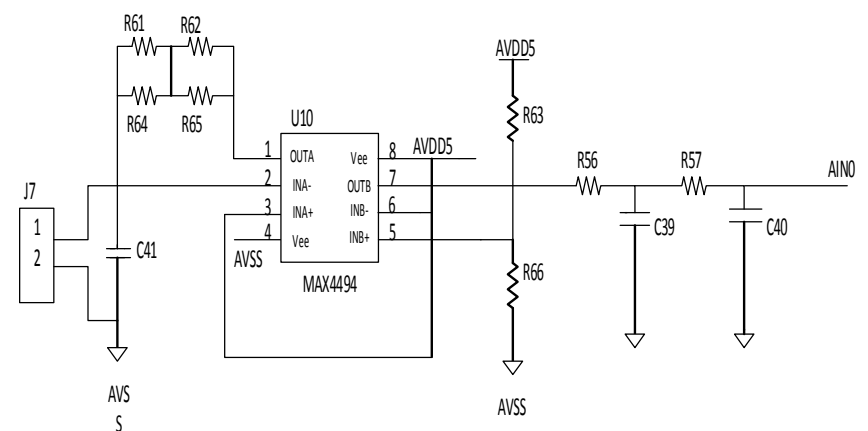

Fig.4 Pressure signal processing circuit

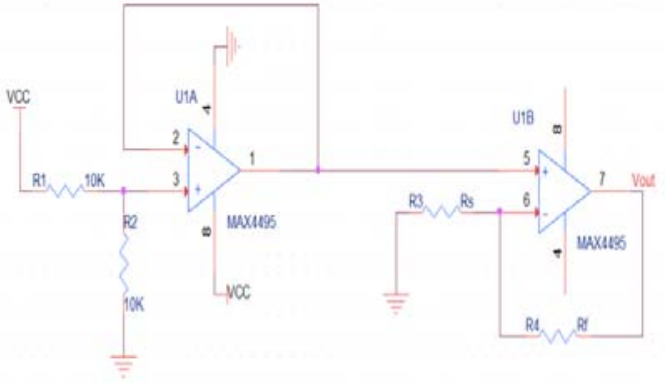

Fig.5 Amplification circuit

The first stage of the MAX4494 plays the role of voltage stabilization and to prevent it from week current injection, which will results in lower accuracy. Thus, the second stage of amplifier can be seen as a single one to work, as formula (1) shows. And the Vout will be reported to the upper-module for processing and storage.

$$
R \mathrm{~s}=\frac{V C C}{2 V_{\text {out }}-V C C} R f
$$

\section{Calibrating Technology of Sensor}

To make sure the ability of the output signal from the flexible sensor, the calibration of sensor is required. In fact, the flexible sensor is charactrized by great number of sensitive points and each sensor system has hundreds of sensing units according demand of its application field. Considering the safety and the stability for electric power grid, adjustmet of all sensitve points is of great importance. Furrthermore, the calibration of sensors must be re-conducted periodelly.

The procedure of sensor calibration is given in Fig.6 in details. This system starts to work from collection elements in sensor of lower module, and analoge signal will be changed into digial one by $\mathrm{AD}$ for reporting to the upper module. And the later one will store received data and display it. By comparing processed data with calibration one, the alarm will be triggered if the pressure or temporature exceed the threshold value, otherwise, a new cycle of monitoring will restart.

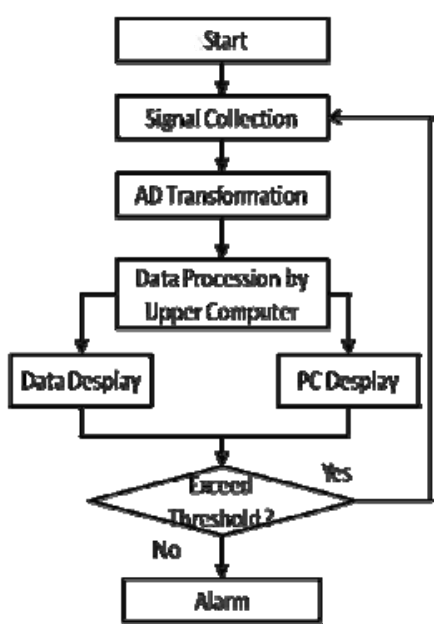


Fig.6 Precedure of Sensor Calibration

\section{Testing Evaluation}

To evaluate the real-time accuracy of the proposed approach, a testing platform is build. In this test, the data collection of three temprature elements and one pressure element is conductled with the frequency of once per second.

The Fig. 7 shows the testing result of temprature signals, where three temprature curves (T13, T15 and T11) represente testing results of three positions on the same battary at the same time. It it is easy to find that the deviation of there 3 curves is small enough to detect current temprature of the battery with high accuracy, and it meets the demand for the sensor to perform temperature detection.

The testing result of pressure signal is given in Fig.8. It can be observed that the value is zero without any pressure and the system is able to notice fastly the pressure signal when pressure is upon the surface of the battery. As various pressure change, the detected value of pressure is also variable in time, which can greatly satisfy demand for the sensor to perform pressure detection.

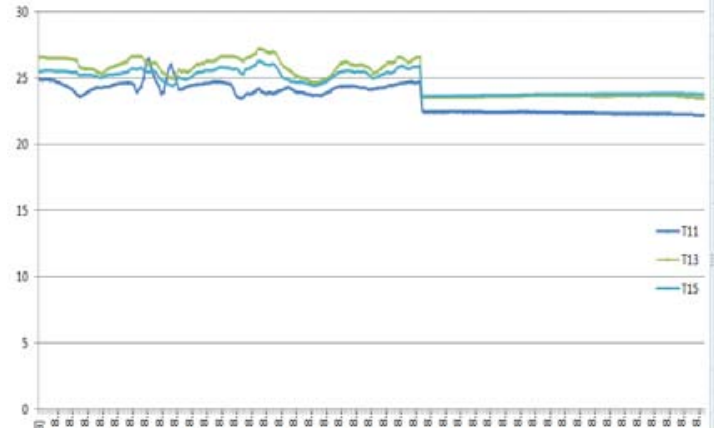

Fig.7 Temprature Signal Curve
F11

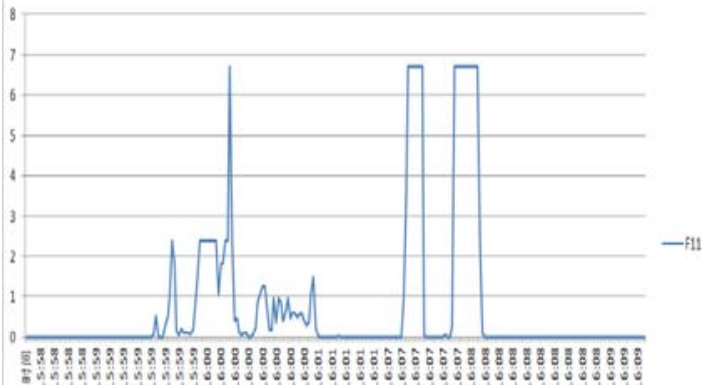

Fig.8 Pressure Signal Curve

\section{Conclusions}

This article proposed a novel signal detection technology for flexible sensor to monitor the temperature and pressure of the battery. The function architecture and the working principle of the flexible sensor was firstly depicted in this work. And the related processing circuits of both temprature and pressure signals were designed. And the Calibrating Technology of Sensor was realized, followed by analysis of detected signal. The testing result showed that the proposed monitoring system was able to perform real-time pressure and temperature detection and pre-alarm with high accuracy and efficiency. Additionally, the efficiency and life-span of battery could be improved greatly with lower cost.

\section{References}

[1] Zhang jiaxing, Chen xiaohui. Journal of electronic measurement and instrument. 2014(02): p.177-183. (in Chinese)

[2] Zhao xiaowen, Design and experiment of signal acquisition system based on LabVIEW[N],2012, 4. (in Chinese)

[3] Xu zhansheng, Yan gaizhen. Power technology. 2013,37,569 571. (in Chinese)

[4] Zheng fuzhong. Study on the flexible sensor based on suspended single wall carbon nanotube array.2011, Chongqing University. (in Chinese)

[5] Yang min,Chen hong. Spacecraft environment engineering. 2009(S1): p. 112-115. (in Chinese)

[6] Guo xiaohui. Journal of electronic measurement and instrument. 2014(11): p. 1254-1261. (in Chinese) 
[7] Chen jie. Monitoring system of intelligent lead acid storage battery[N], Mechanical and electrical engineering,1999(06). (in Chinese) 